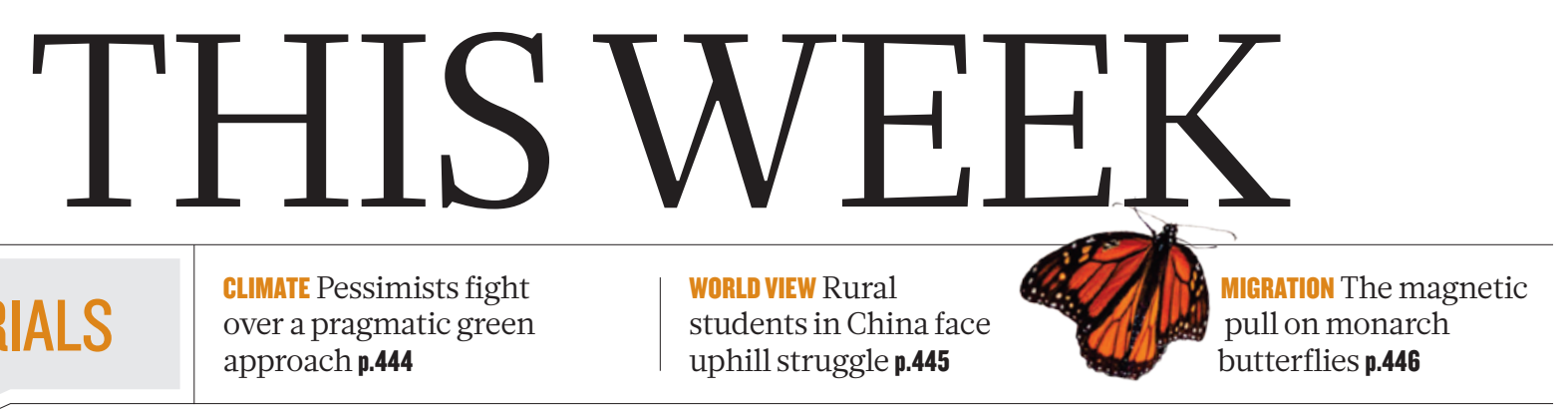

\title{
Biosafety in the balance
}

\author{
An accident with anthrax demonstrates that pathogen research always carries a risk of release - \\ and highlights the need for rigorous scrutiny of gain-of-function flu studies.
}

$\mathrm{T}$ The news last week of an accident involving live anthrax bacteria at the US Centers for Disease Control and Prevention (CDC) in Atlanta, Georgia, is troubling. Some 84 workers were potentially exposed to the deadly Ames strain at three CDC labs. But the incident will cause much wider ripples: it highlights the risks of the current proliferation of biocontainment labs and work on dangerous pathogens. If an accident can happen at the CDC, then it can happen anywhere.

Details are sparse, but it seems that the anthrax was being inactivated in a biosafety-level-3 (BSL-3) high-containment lab so that it could be studied at the three BSL-2 labs. But live bacteria survived the inactivation step, and were not detected before samples were sent out. The CDC considers the risk that the exposed workers have been infected to be low, and all have been offered protective antibiotics.

Such lab accidents are fortunately not commonplace. A CDC analysis in 2012 reported, for example, that there were 727 incidents of theft, loss or release of Select Agents and Toxins in the United States between 2004 and 2010, resulting in 11 laboratory-acquired infections and no secondary transmission (R. D. Henkel et al. Appl. Biosafety 17, $171-180 ; 2012)$. Anthrax is contracted by direct exposure to spores, and does not spread between people. Much more potentially dangerous are lab accidents involving agents that do. It is impossible to read about the CDC incident and not breathe a large sigh of relief that it did not involve a novel engineered pandemic influenza strain.

Groups led by Ron Fouchier of the Erasmus Medical Center in Rotterdam, the Netherlands, and Yoshihiro Kawaoka of the University of Wisconsin-Madison created a storm in late 2011 when they artificially engineered potentially pandemic forms of the $\mathrm{H} 5 \mathrm{~N} 1$ avian flu virus. In January last year, researchers ended a voluntary 12-month moratorium on such gain-of-function flu research, which can increase the host range, transmissibility or virulence of viruses (see Nature 493, 460; 2013), and work resumed.

This month, Kawaoka's group reported that it had engineered a de novo flu virus from wild-avian-flu-strain genes that coded for proteins similar to those in the 1918 pandemic virus (T. Watanabe Cell Host Microbe 15, 692-705; 2014). The researchers were able to make a virulent version that could transmit between ferrets, and they concluded that a 1918-like virus could therefore emerge from wild avian flu viruses.

In the century since the 1918 flu hit, no similar pandemic variant has emerged despite wild animal flu viruses mutating and reassorting incessantly. The $1918 \mathrm{H} 1 \mathrm{~N} 1$ virus was reconstructed in 2005, but human immunity to it became widespread following the $2009 \mathrm{H} 1 \mathrm{~N} 1$ pandemic. There are no mammalian-transmissible 1918-like avian flus in the wild; the only ones that exist are Kawaoka's team's engineered strains.

Researchers such as Kawaoka and Fouchier argue that by engineering mutant viruses in the lab, they can identify mutations and traits that allow the pathogens to spread between mammals. This in turn, they argue, allows assessment of the pandemic potential of animal-flu viruses. In the long term, such experiments could help to elucidate the mechanisms of virus transmissibility and pathogenicity. But their shorter-term public-health benefits have been overstated. The risks and benefits must therefore be carefully weighed, and rigorous oversight is needed to ensure that such work is done only at facilities with the highest standards of biosafety.

Other scientists argue that the concept of predicting the pandemic potential of flu viruses from mutations, although appealing, is simplis-

"The idea of
an accidental
release of a
potentially
pandemic flu
virus cannot
be completely
written off."
tic. They say that the identified mutations are but a handful out of millions of possible combinations, many of which might also allow mammalian transmission. They argue that mutations in specific proteins cannot reliably predict traits, and that outcomes depend on interactions between various other background genetic changes throughout the virus.

These points were highlighted in a paper in PLoS Medicine last month (M. Lipsitch and A. P. Galvani PLoS Med. 11, e1001646; 2014), and in a letter by 56 leading virologists, infectious-disease specialists and public-health experts to European Commission president José Manuel Barroso last December (see Nature http://doi.org/tdb; 2013). They also question the claimed public-health benefits of such research, and argue that similar information could be obtained through safer experiments. Opponents of gain-of-function flu research call, in particular, for more rigorous risk-benefit assessments. The CDC accident shows that, should such research proliferate, the idea of an accidental release of a potentially pandemic flu virus cannot be completely written off. This demands that such research proposals receive the utmost scrutiny.

A US Government Accountability Office report released in February last year expressed concern that the proliferation of US high-containment labs following the terrorist attacks of 11 September 2001 and the anthrax-letter attacks the same year was proceeding without a rigorous assessment of the nation's real needs across all government agencies, universities and private companies. "Increasing the number of laboratories also increases the aggregate national risk," it noted. No one keeps track, for example, of how many BSL-3 labs there are in the United States alone, although their number is thought to be in the thousands. The number of such labs is increasing in China and elsewhere.

After smallpox was eradicated in 1980, there was a concerted international effort to reduce the number of labs holding stocks to just two: one at the CDC and one at the Russian State Research Center of Virology and Biotechnology in Koltsovo. All research at these centres must be approved by the World Health Organization. The fewer the labs that perform experiments, the smaller is the risk of an accidental release. But as the CDC accident reminds us, should gain-of-function flu research proliferate, in particular at facilities with less than exemplary biosafety standards, the risks of an accidental release of a potentially pandemic flu virus will be multiplied. 